\title{
Energy-weighted sum rules for mesons in hot and dense matter
}

\author{
D. Cabrera, ${ }^{1}$ A. Polls, ${ }^{2}$ A. Ramos, ${ }^{2}$ and L. Tolós ${ }^{3}$ \\ ${ }^{1}$ Departamento de Física Teórica II, Universidad Complutense, 28040 Madrid, Spain \\ ${ }^{2}$ Departament d'Estructura i Constituents de la Matèria, Universitat de Barcelona, Diagonal 647, 08028 Barcelona, Spain \\ ${ }^{3}$ Theory Group, KVI, University of Groningen, Zernikelaan 25, 9747 AA Groningen, The Netherlands
}

(Received 10 March 2009; published 6 October 2009)

\begin{abstract}
We study energy-weighted sum rules of the pion and kaon propagator in nuclear matter at finite temperature. The sum rules are obtained from matching the Dyson form of the meson propagator with its spectral Lehmann representation at low and high energies. We calculate the sum rules for specific models of the kaon and pion self-energy. The in-medium spectral densities of the $K$ and $\bar{K}$ mesons are obtained from a chiral unitary approach in coupled channels that incorporates the $S$ and $P$ waves of the kaon-nucleon interaction. The pion self-energy is determined from the $P$-wave coupling to particle-hole and $\Delta$-hole excitations, modified by short-range correlations. The sum rules for the lower-energy weights are fulfilled satisfactorily and reflect the contributions from the different quasiparticle and collective modes of the meson spectral function. We discuss the sensitivity of the sum rules to the distribution of spectral strength and their usefulness as quality tests of model calculations.
\end{abstract}

DOI: 10.1103/PhysRevC.80.045201

PACS number(s): 13.75.Gx, 13.75.Jz, 14.40.Aq, 21.65.-f

\section{INTRODUCTION}

The properties of hadrons, both mesons and baryons, in hot and dense matter have been a matter of intense investigations over the past several years [1-4] and it is a subject that is calling the attention of many present and future experimental programs [5].

Of particular relevance are the lightest strange and nonstrange mesons, namely kaons and pions. They typically appear as final-state interacting particles in nuclear production reactions. Being light, these pseudoscalar mesons are also abundantly produced as thermal excitations in heavy-ion collisions. Moreover, they constitute a relevant ingredient in the medium modification of vector $(\rho, \omega, \phi)$ and axial-vector $\left(a_{1}\right)$ mesons, as these decay strongly into light mesons and thus their in-medium properties are tied to the modifications of the meson-cloud component of their spectra. Vector-mesons provide a unique tool to study high-density and/or temperature regions from electromagnetic decays, whereas the combined study of the vector and axial-vector spectral functions can shed light on the onset and physical realization of chiral symmetry restoration in hot/dense strongly interacting matter. Therefore, a solid knowledge of their interactions with the medium through their coupling to light pseudoscalar mesons is mandatory. Another relevant role of the properties of light mesons in nuclear matter is played in the study of mesic atoms and nuclei, where the observation of bound states of mesons and their spectral properties can lead to a better understanding of meson-meson and meson-baryon interactions at finite nuclear density.

One of the aims of many theoretical studies is to describe the propagation of hadrons in hot and dense matter. A natural way to face this problem is to build the single-particle Green's function of the hadron. In turn, the latter requires the knowledge of the hadron self-energy, which describes the interactions of the particle with the medium. Obviously, the quality of the calculation of the self-energy relies on having a good model for the interaction and a robust many-body framework. The single-particle Green's function has welldefined analytical properties that impose some constraints on both the many-body formalism and the interaction model. To exploit these analytical properties it is convenient to introduce the Lehmann representation, which gives the Green's function in terms of the single-particle spectral functions. An excellent tool to analyze these constraints is provided by the energyweighted sum rules (EWSR) of the single-particle spectral functions.

Energy-weighted sum rules have been extensively and successfully used in the literature, mainly to analyze the response function of many-body systems in particular for nuclear matter [6], quantum liquids [7], and, more recently, in the context of cold atoms [8]. The energy-weighted moments of the response to a given operator allow one to estimate the low-energy states excited by this operator, specially for highly collective states that concentrate a substantial amount of strength. An important advantage of the sum rules is that they can be most of the times directly calculated without knowing the response function by just evaluating the expectation value in the ground state of commutators involving the excitation operator and the Hamiltonian [6].

In the context of chiral-symmetry breaking in hadronic physics, one finds a prominent example in the well-known set of EWSR's proposed by Weinberg [9], which has been extended to hot and dense matter systems [10]. The first of these relations connects the integrated difference of the vector and axial vector mesonic spectral functions (current-current correlators) with the pion decay constant. Together with suitable model calculations, the first Weinberg sum rule can shed light on the degree and physical mechanism of chiral symmetry restoration at finite nuclear densities [11,12].

In the case of single-particle Green's functions, EWSR's for the nucleon spectral functions have been well established for a long time in the literature [13]. However, only recently has the progress in the numerical calculation of the single-particle spectral functions in nuclear matter permitted, through a careful analysis of the EWSR's, identification of the effects 
of nucleon-nucleon correlations in the distribution of the single-particle strength, both at zero [14] and finite temperature $[15,16]$.

However, EWSR's have not been used much for the case of meson single-particle properties. They have been obtained in Ref. [17] for $\omega$ mesons coupling to particle-hole excitations in a dense medium within the long-wavelength approximation. In this article, we present a derivation of the EWSR for the single-particle spectral functions associated to the propagation of mesons in a hot and dense medium and discuss the physical implications of the fulfillment of these sum rules in connection with the underlying interaction models as well as with certain aspects of the meson nuclear phenomenology. Our aim is not only to analyze the consistency of the many-body formalism used to calculate the meson Green's function but also to obtain useful insights on the validity of the meson-nucleon interaction model.

In Sec. II we discuss the derivation of the EWSR's for mesons propagating in cold nuclear matter and particularize for the case of kaons and pions. In the case of kaons, the particle $(K)$ and antiparticle $(\bar{K})$ modes behave differently in the nuclear medium, while the isotriplet pions exhibit a common behavior in symmetric nuclear matter, which allows for a simplification in the sum rules expressions. The generalization of the sum rules to nuclear matter at finite temperature is also provided in this section. In Sec. III we briefly summarize particular models of the kaon and pion self-energies in the nuclear medium, which have been discussed elsewhere. The kaon self-energy is built from the effective kaon nucleon interaction in $S$ and $P$ waves described in a chiral unitary approach $[18,19]$. In the case of pions, we consider the standard $P$-wave coupling to $\mathrm{ph}$ and $\Delta \mathrm{h}$ configurations modified by spin-isospin short-range correlations [20,21]. The resulting sum rules for kaons and pions are discussed in Sec. IV, for various momenta, nuclear densities, and temperatures. We analyze the contribution of the different collective modes by studying the saturation of the sum rules as a function of the energy and discuss useful insights that can be drawn for the particular self-energy models. A summary of our main conclusions is presented in Sec. V, together with some discussion on the application of the present method to test the consistency of the many-body scheme or the meson nuclear interaction model in a hot symmetric nuclear medium, as well as in other scenarios that will be explored in the near future.

\section{DERIVATION OF ENERGY-WEIGHTED SUM RULES}

\section{A. Zero temperature}

The derivation of the energy-weighted sum rules (EWSRs) for hadrons and, in particular, for mesons follows from comparing the in-medium propagator with the corresponding Lehmann spectral representation. The propagator for a meson $M$ of energy $q^{0}$ and momentum $\vec{q}$ in symmetric nuclear matter of density $\rho$ reads:

$$
D_{M}\left(q^{0}, \vec{q} ; \rho\right)=\frac{1}{\left(q^{0}\right)^{2}-\omega_{M}^{2}(\vec{q})-\Pi_{M}\left(q^{0}, \vec{q} ; \rho\right)},
$$

where $\omega_{M}(\vec{q})=\sqrt{m^{2}+\vec{q}^{2}}$ is the free dispersion relation and $\Pi_{M}$ the meson self-energy. The corresponding spectral (Lehmann) representation when the meson and antimeson behave as distinct particles in the medium is

$$
D_{M}\left(q^{0}, \vec{q} ; \rho\right)=\int_{0}^{\infty} d \omega\left\{\frac{S_{M}(\omega, \vec{q} ; \rho)}{q^{0}-\omega+\mathrm{i} \eta}-\frac{S_{\bar{M}}(\omega, \vec{q} ; \rho)}{q^{0}+\omega+\mathrm{i} \eta}\right\}
$$

with

$$
S_{M}\left(q^{0}, \vec{q} ; \rho\right)=-\frac{1}{\pi} \operatorname{Im} D_{M}\left(q^{0}, \vec{q} ; \rho\right) .
$$

We start by analyzing the $q^{0} \rightarrow \infty$ limit. To obtain the expansion of the propagator in powers of $1 / q^{0}$ we first study the behavior of the self-energy $\Pi_{M}\left(q^{0}, \vec{q} ; \rho\right)$ at high energies from its dispersion relation

$$
\Pi_{M}\left(q^{0}, \vec{q} ; \rho\right)=\Pi_{M}^{\infty}(\vec{q} ; \rho)-\frac{1}{\pi} \int_{-\infty}^{\infty} d \omega \frac{\operatorname{Im} \Pi_{M}(\omega, \vec{q} ; \rho)}{q^{0}-\omega+i \eta},
$$

where $\Pi_{M}^{\infty}$ is the real nondispersive contribution of the selfenergy. In the particular models discussed in the next section, this quantity will be either zero or stay finite. By expanding the real part of Eq. (4) around $q^{0} \rightarrow \infty$ we obtain:

$$
\begin{aligned}
\operatorname{Re} & \Pi_{M}\left(q^{0}, \vec{q} ; \rho\right) \\
= & \Pi_{M}^{\infty}(\vec{q} ; \rho)-\frac{1}{\pi} \frac{1}{q^{0}}\left[\int_{-\infty}^{\infty} d \omega \operatorname{Im} \Pi_{M}(\omega, \vec{q} ; \rho)\right. \\
& +\frac{1}{q^{0}} \int_{-\infty}^{\infty} d \omega \omega \operatorname{Im} \Pi_{M}(\omega, \vec{q} ; \rho) \\
& \left.+\frac{1}{\left(q^{0}\right)^{2}} \int_{-\infty}^{\infty} d \omega \omega^{2} \operatorname{Im} \Pi_{M}(\omega, \vec{q} ; \rho)+\cdots\right] .
\end{aligned}
$$

Using the properties of the retarded self-energy:

$$
\begin{aligned}
& \operatorname{Re} \Pi_{M}\left(-q^{0}, \vec{q} ; \rho\right)=\operatorname{Re} \Pi_{\bar{M}}\left(q^{0}, \vec{q} ; \rho\right) \\
& \operatorname{Im} \Pi_{M}\left(-q^{0}, \vec{q} ; \rho\right)=-\operatorname{Im} \Pi_{\bar{M}}\left(q^{0}, \vec{q} ; \rho\right)
\end{aligned}
$$

we can rewrite Eq. (5) as

$$
\begin{aligned}
\operatorname{Re} & \Pi_{M}\left(q^{0}, \vec{q} ; \rho\right) \\
= & \Pi_{M}^{\infty}(\vec{q} ; \rho)-\frac{1}{\pi} \frac{1}{q^{0}}\left\{\int _ { 0 } ^ { \infty } d \omega \left[\operatorname{Im} \Pi_{M}(\omega, \vec{q} ; \rho)\right.\right. \\
& \left.\quad-\operatorname{Im} \Pi_{\bar{M}}(\omega, \vec{q} ; \rho)\right]+\frac{1}{q^{0}} \int_{0}^{\infty} d \omega \omega\left[\operatorname{Im} \Pi_{M}(\omega, \vec{q} ; \rho)\right. \\
& \left.+\operatorname{Im} \Pi_{\bar{M}}(\omega, \vec{q} ; \rho)\right]+\frac{1}{\left(q^{0}\right)^{2}} \int_{0}^{\infty} d \omega \omega^{2}\left[\operatorname{Im} \Pi_{M}(\omega, \vec{q} ; \rho)\right. \\
& \left.\left.-\operatorname{Im} \Pi_{\bar{M}}(\omega, \vec{q} ; \rho)\right]+\cdots\right\} .
\end{aligned}
$$

Accordingly, the first few terms of the expansion of the real part of the in-medium propagator [Eq. (1)] read:

$$
\begin{aligned}
\operatorname{Re} & D_{M}\left(q^{0}, \vec{q} ; \rho\right) \\
= & \frac{1}{\left(q^{0}\right)^{2}}\left\{1+\frac{1}{\left(q^{0}\right)^{2}}\left[\omega_{M}^{2}(\vec{q})+\Pi_{M}^{\infty}(\vec{q} ; \rho)\right]\right. \\
& -\frac{1}{\pi} \frac{1}{\left(q^{0}\right)^{3}} \int_{0}^{\infty} d \omega\left[\operatorname{Im} \Pi_{M}(\omega, \vec{q} ; \rho)-\operatorname{Im} \Pi_{\bar{M}}(\omega, \vec{q} ; \rho)\right]
\end{aligned}
$$




$$
\begin{aligned}
& +\frac{1}{\left(q^{0}\right)^{4}}\left(\left[\omega_{M}^{2}(\vec{q})+\Pi_{M}^{\infty}(\vec{q} ; \rho)\right]^{2}\right. \\
& \left.-\frac{1}{\pi} \int_{0}^{\infty} d \omega \omega\left[\operatorname{Im} \Pi_{M}(\omega, \vec{q} ; \rho)+\operatorname{Im} \Pi_{\bar{M}}(\omega, \vec{q} ; \rho)\right]\right) \\
& +\cdots\}
\end{aligned}
$$

However, we obtain the following expansion around $q^{0} \rightarrow \infty$ from the Lehmann representation [Eq. (2)]:

$$
\begin{aligned}
\operatorname{Re} & D_{M}\left(q^{0}, \vec{q} ; \rho\right) \\
= & \frac{1}{q^{0}} \sum_{n=0}^{\infty} \int_{0}^{\infty} d \omega\left[\frac{\omega}{q^{0}}\right]^{2 n}\left[S_{M}(\omega, \vec{q} ; \rho)-S_{\bar{M}}(\omega, \vec{q} ; \rho)\right] \\
& +\frac{1}{q^{0}} \sum_{m=0}^{\infty} \int_{0}^{\infty} d \omega\left[\frac{\omega}{q^{0}}\right]^{2 m+1}\left[S_{M}(\omega, \vec{q} ; \rho)+S_{\bar{M}}(\omega, \vec{q} ; \rho)\right],
\end{aligned}
$$

which displays separately the terms involving the sum and the difference of the meson and antimeson spectral functions. The sum rules are readily obtained from matching Eqs. (8) and (9) order by order in $1 / q^{0}$. The first few terms up to $\left(1 / q^{0}\right)^{4}$ determine:

$$
\begin{aligned}
& m_{0}^{(\mp)}(q ; \rho): \\
& (n=0) \int_{0}^{\infty} d \omega\left[S_{M}(\omega, \vec{q} ; \rho)-S_{\bar{M}}(\omega, \vec{q} ; \rho)\right]=0 \\
& (m=0) \int_{0}^{\infty} d \omega \omega\left[S_{M}(\omega, \vec{q} ; \rho)+S_{\bar{M}}(\omega, \vec{q} ; \rho)\right]=1 \\
& m_{1}^{(\mp)}(q ; \rho): \\
& (n=1) \int_{0}^{\infty} d \omega \omega^{2}\left[S_{M}(\omega, \vec{q} ; \rho)-S_{\bar{M}}(\omega, \vec{q} ; \rho)\right]=0 \\
& (m=1) \int_{0}^{\infty} d \omega \omega^{3}\left[S_{M}(\omega, \vec{q} ; \rho)+S_{\bar{M}}(\omega, \vec{q} ; \rho)\right] \\
& \quad=\omega_{M}^{2}(\vec{q})+\Pi_{M}^{\infty}(\vec{q} ; \rho) .
\end{aligned}
$$

The dispersive part of the self-energy contributes to the righthand side starting from $\left(1 / q^{0}\right)^{5}$. For instance, in the case $n=$ $m=2$ the sum rules read:

$$
\begin{aligned}
& m_{2}^{(\mp)}(q ; \rho): \\
& (n=2) \int_{0}^{\infty} d \omega \omega^{4}\left[S_{M}(\omega, \vec{q} ; \rho)-S_{\bar{M}}(\omega, \vec{q} ; \rho)\right] \\
& =-\frac{1}{\pi} \int_{0}^{\infty} d \omega\left[\operatorname{Im} \Pi_{M}(\omega, \vec{q} ; \rho)-\operatorname{Im} \Pi_{\bar{M}}(\omega, \vec{q} ; \rho)\right] \\
& (m=2) \int_{0}^{\infty} d \omega \omega^{5}\left[S_{M}(\omega, \vec{q} ; \rho)+S_{\bar{M}}(\omega, \vec{q} ; \rho)\right] \\
& =\left[\omega_{M}^{2}(\vec{q})+\Pi_{M}^{\infty}(\vec{q} ; \rho)\right]^{2}-\frac{1}{\pi} \int_{0}^{\infty} d \omega \omega\left[\operatorname{Im} \Pi_{M}(\omega, \vec{q} ; \rho)\right. \\
& \left.\quad+\operatorname{Im} \Pi_{\bar{M}}(\omega, \vec{q} ; \rho)\right] .
\end{aligned}
$$

Furthermore, another sum rule results from the evaluation of the zero-mode propagator, namely $q^{0}=0$ :

$$
\begin{aligned}
& m_{-1}(q ; \rho): \int_{0}^{\infty} d \omega \frac{1}{\omega}\left[S_{M}(\omega, \vec{q} ; \rho)+S_{\bar{M}}(\omega, \vec{q} ; \rho)\right] \\
& =\frac{1}{\omega_{M}^{2}(\vec{q})+\Pi_{M}(0, \vec{q} ; \rho)} .
\end{aligned}
$$

Note that, as implied by the sum rules $m_{-1}$ and $m_{1}^{(+)}$, the selfenergy $\Pi_{M}\left(q^{0}, \vec{q} ; \rho\right)$ at $q^{0}=0$ and $q^{0} \rightarrow \infty$ is necessarily real. One expects this from the phenomenological point of view: at zero energy there should not be any open in-medium channel for the meson to decay into, whereas at high energies form factors or cutoffs are usually applied to truncate the modes not accounted for explicitly by the hadronic model. Also note that the sum rules have to be satisfied for every value of the meson momentum, $q$. The $m_{0}$ sum rule is of particular relevance, because it is a consequence of the canonical commutation relation of the meson field [22].

The sum rules given by Eqs. (10) to (16) are valid for the general case in which the meson and the antimeson behave differently in the nuclear medium, such as kaons and antikaons, or pions in asymmetric nuclear matter. For instance, by substituting $M \rightarrow \bar{K}$ and $\bar{M} \rightarrow K$ the former expressions would represent the sum rules for the antikaon. Similar expressions would be obtained for the kaon case if $M \rightarrow K$ and $\bar{M} \rightarrow \bar{K}$. It is worth noting that, even if the $\bar{K} N$ and $K N$ interactions are very different in nuclear matter, the EWSRs impose constraints on the behavior of the $\bar{K}$ and $K$ self-energies. In particular and due to the symmetry under the exchange $K \leftrightarrow \bar{K}$ on the left-hand side, the $m_{-1}$ and $m_{1}^{(+)}$sum rules indicate that not only $\Pi_{\bar{K}}\left(q^{0}, \vec{q}\right)$ and $\Pi_{K}\left(q^{0}, \vec{q}\right)$ should be real for $q^{0}=0$ and $q^{0} \rightarrow \infty$ but also that both must coincide at the corresponding low- and high-energy limits. Actually, this is a consequence of the crossing symmetry relations given in Eq. (6). Therefore, sum rules obtained from models that do not respect this symmetry will be fulfilled only to a certain level, depending on the severity of the violation.

In the particular case of pions in symmetric matter, we have $\Pi_{M}\left(q^{0}, \vec{q} ; \rho\right)=\Pi_{\bar{M}}\left(q^{0}, \vec{q} ; \rho\right)$ because particles and antiparticles behave identically. Consequently, only the even powers of $1 / q^{0}$ survive in the expansion of the propagator and, correspondingly, the sum rules acquire the following simplified forms:

$$
\begin{aligned}
& m_{0}(q ; \rho): \int_{0}^{\infty} d \omega 2 \omega S_{\pi}(\omega, \vec{q} ; \rho)=1 \\
& m_{1}(q ; \rho): \int_{0}^{\infty} d \omega 2 \omega^{3} S_{\pi}(\omega, \vec{q} ; \rho)=\omega_{\pi}^{2}(\vec{q})+\Pi_{\pi}^{\infty}(\vec{q} ; \rho) \\
& m_{-1}(q ; \rho): \int_{0}^{\infty} d \omega \frac{2}{\omega} S_{\pi}(\omega, \vec{q} ; \rho)=\frac{1}{\omega_{\pi}^{2}(\vec{q})+\Pi_{\pi}(0, \vec{q} ; \rho)} .
\end{aligned}
$$

\section{B. Finite temperature}

The extension of the EWSRs to finite temperature $T$ is straightforward. We elaborate on this for the antikaon 
case below, whereas for the pion case the derivation is completely similar. Once again, the sum rules are obtained from the expansion at high energy of both the Dyson form of the propagator and its Lehmann representation. At finite $T$, the spectral representation is obtained in the Matsubara space, namely

$$
D_{\bar{K}}\left(\omega_{n}, \vec{q} ; \rho, T\right)=-\frac{1}{\pi} \int_{-\infty}^{\infty} d \omega \frac{\operatorname{Im} D_{\bar{K}}(\omega, \vec{q} ; \rho, T)}{\mathrm{i} \omega_{n}-\omega},
$$

where $\mathrm{i} \omega_{n}=2 n \pi T$ is the bosonic Matsubara frequency. If we now split the integral in two pieces,

$$
\begin{aligned}
D_{\bar{K}}\left(\omega_{n}, \vec{q} ; \rho, T\right)= & -\frac{1}{\pi}\left[\int_{-\infty}^{0} d \omega \frac{\operatorname{Im} D_{\bar{K}}(\omega, \vec{q} ; \rho, T)}{\mathrm{i} \omega_{n}-\omega}\right. \\
& \left.+\int_{0}^{\infty} d \omega \frac{\operatorname{Im} D_{\bar{K}}(\omega, \vec{q} ; \rho, T)}{\mathrm{i} \omega_{n}-\omega}\right]
\end{aligned}
$$

and change $\omega \rightarrow-\omega$ in the first term, we obtain

$$
\begin{aligned}
D_{\bar{K}}\left(\omega_{n}, \vec{q} ; \rho, T\right)= & -\frac{1}{\pi}\left[\int_{0}^{\infty} d \omega \frac{\operatorname{Im} D_{\bar{K}}(-\omega, \vec{q} ; \rho, T)}{\mathrm{i} \omega_{n}+\omega}\right. \\
& \left.+\int_{0}^{\infty} d \omega \frac{\operatorname{Im} D_{\bar{K}}(\omega, \vec{q} ; \rho, T)}{\mathrm{i} \omega_{n}-\omega}\right] .
\end{aligned}
$$

In a fully relativistic thermal calculation, the imaginary part of the retarded self-energy satisfies

$$
\operatorname{Im} \Pi_{\bar{K}}(-\omega, \vec{q} ; \rho, T)=-\operatorname{Im} \Pi_{K}(\omega, \vec{q} ; \rho, T)
$$

and, hence, the same applies to the spectral function. Thus, the spectral representation of the propagator actually reads

$$
\begin{aligned}
D_{\bar{K}}\left(\omega_{n}, \vec{q} ; \rho, T\right)= & -\frac{1}{\pi}\left[\int_{0}^{\infty} d \omega \frac{\operatorname{Im} D_{\bar{K}}(\omega, \vec{q} ; \rho, T)}{\mathrm{i} \omega_{n}-\omega}\right. \\
& \left.-\int_{0}^{\infty} d \omega \frac{\operatorname{Im} D_{K}(\omega, \vec{q} ; \rho, T)}{\mathrm{i} \omega_{n}+\omega}\right] .
\end{aligned}
$$

We then perform the analytical continuation onto the real axis, $\mathrm{i} \omega_{n} \rightarrow q^{0}+\mathrm{i} \eta$, and one finally gets

$$
\begin{aligned}
& D_{\bar{K}}\left(q^{0}, \vec{q} ; \rho, T\right) \\
& \quad=\int_{0}^{\infty} d \omega\left\{\frac{S_{\bar{K}}(\omega, \vec{q} ; \rho, T)}{q^{0}-\omega+\mathrm{i} \eta}-\frac{S_{K}(\omega, \vec{q} ; \rho, T)}{q^{0}+\omega+\mathrm{i} \eta}\right\},
\end{aligned}
$$

where

$$
S_{\bar{K}(K)}(\omega, \vec{q} ; \rho, T)=-\frac{1}{\pi} \operatorname{Im} D_{\bar{K}(K)}(\omega, \vec{q} ; \rho, T) .
$$

The expression of Eq. (25) for the spectral representation of the propagator has the same behavior at $q^{0}=0$ and $q^{0} \rightarrow \infty$ as the one obtained in the $T=0$ case. Therefore, the EWSRs at finite density and temperature have the same form as the ones at $T=0$. Summarizing, for $m_{-1}, m_{0}^{(\mp)}$, and $m_{1}^{(\mp)}$ one has

$$
\begin{aligned}
& m_{-1}(q ; \rho, T): \int_{0}^{\infty} d \omega \frac{1}{\omega}\left[S_{\bar{K}}(\omega, \vec{q} ; \rho, T)+S_{K}(\omega, \vec{q} ; \rho, T)\right] \\
& =\frac{1}{\omega_{K}^{2}(\vec{q})+\Pi_{\bar{K}}(0, \vec{q} ; \rho, T)}
\end{aligned}
$$

$$
\begin{aligned}
m_{0}^{(\mp)}(q ; \rho, T): & \int_{0}^{\infty} d \omega\left[S_{\bar{K}}(\omega, \vec{q} \rho, T)-S_{K}(\omega, \vec{q} ; \rho, T)\right]=0 \\
& \int_{0}^{\infty} d \omega \omega\left[S_{\bar{K}}(\omega, \vec{q} ; \rho, T)+S_{K}(\omega, \vec{q} ; \rho, T)\right]=1
\end{aligned}
$$

$$
\begin{gathered}
m_{1}^{(\mp)}(q ; \rho, T): \int_{0}^{\infty} d \omega \omega^{2}\left[S_{\bar{K}}(\omega, \vec{q} ; \rho, T)-S_{K}(\omega, \vec{q} ; \rho, T)\right] \\
=0 \\
\int_{0}^{\infty} d \omega \omega^{3}\left[S_{\bar{K}}(\omega, \vec{q} ; \rho, T)+S_{K}(\omega, \vec{q} ; \rho, T)\right] \\
=\omega_{K}^{2}(\vec{q})+\Pi_{\bar{K}}^{\infty}(\vec{q} ; \rho, T) .
\end{gathered}
$$

\section{III. $\bar{K}, K$, AND PION SELF-ENERGY MODELS}

The EWSRs constitute an ideal test of the quality of any hadronic model. The energy-weighted integrals of the hadronic spectral function, on the one side, are compared to the lowand high-energy limits of the corresponding self-energy or to model-independent values, on the other side.

Here we briefly recall the essential features of recent calculations of the properties of kaons in dense matter at zero and finite temperature. We refer to Refs. [18,19] for details. The $\bar{K}$ and $K$ self-energies in symmetric nuclear matter at finite temperature are obtained from an evaluation of the in-medium kaon-nucleon interaction within a chiral unitary approach. The model incorporates the $S$ and $P$ waves of the kaon-nucleon interaction.

At tree level, the $S$-wave amplitude arises from the Weinberg-Tomozawa term of the chiral Lagrangian. Unitarization in coupled channels is imposed by solving the Bethe-Salpeter equation with on-shell amplitudes. With a single regularization parameter, the unitarized $\bar{K} N$ amplitude generates dynamically the $\Lambda(1405)$ resonance in the $I=0$ channel and provides a satisfactory description of low-energy scattering observables. The in-medium solution of the $S$-wave amplitude accounts for Pauli-blocking effects, mean-field binding on the nucleons and hyperons via a $\sigma-\omega$ model, and the dressing of the pion and kaon propagators through their corresponding self-energies in a self-consistent manner. The relation

$$
\begin{aligned}
\Pi_{\bar{K}(K)}^{s_{(}}\left(q_{0}, \vec{q} ; \rho, T\right)= & \int \frac{d^{3} p}{(2 \pi)^{3}} n_{N}(\vec{p}, T)\left[T_{\bar{K}(K) N}^{(I=0)}\left(P_{0}, \vec{P} ; \rho, T\right)\right. \\
& \left.+3 T_{\bar{K}(K) N}^{(I=1)}\left(P_{0}, \vec{P} ; \rho, T\right)\right]
\end{aligned}
$$

determines the antikaon (kaon) dominant $S$-wave component of the self-energy in terms of the in-medium effective antikaon(kaon)-nucleon interaction in the $S$ wave.

We should mention that the loop integrals are regularized by a cutoff momentum of $q_{\max }=630 \mathrm{MeV} / c$. This means that the right-hand side unitary cut is correctly implemented up to center-of-mass energies $\sqrt{s}$ of about $2 \mathrm{GeV}$, above which the model cannot be trusted. This in turn imposes a limit of $q^{0} \sim$ $1 \mathrm{GeV}$ for the calculated self-energies of the $K$ and $\bar{K}$ mesons. We will make sure in the next section that energies beyond this range no longer contribute to the sum rule under study. The model incorporates, in addition, a $P$-wave contribution to the 
self-energy from hyperon-hole (Yh) excitations, including $\Lambda$, $\Sigma$, and $\Sigma^{*}$ components.

Finite temperature effects are implemented in the intermediate meson-baryon states following the imaginary time formalism, thus keeping the analytical constraints of the retarded self-energies of the $K$ and $\bar{K}$ mesons.

The results from Ref. [19] show that the $\bar{K}$ effective mass gets lowered by about $50 \mathrm{MeV}$ in cold nuclear matter at saturation density, whereas finite temperature reduces this attraction to $50 \%$ at $T=100 \mathrm{MeV}$. The $P$-wave contribution to the $\bar{K}$ optical potential, due to $\Lambda, \Sigma$, and $\Sigma^{*}$ excitations, becomes significant for momenta larger than $200 \mathrm{MeV} / c$ and softens the attraction felt by the $\bar{K}$ in the nuclear medium moderately. The $\bar{K}$ spectral function spreads over a wide range of energies, reflecting the melting of the $\Lambda(1405)$ resonance and the Yh contributions at finite temperature. Regarding the $K$ self-energy, it is found that the low-density theorem is a good approximation close to saturation density, due to the absence of resonance-hole excitations in the $K N$ interaction. The $K$ potential shows a moderate repulsive behavior, whereas the quasiparticle peak is considerably broadened with increasing density and temperature. Implications of these results for the decay of the $\phi$ meson and transport calculations in heavy-ion collisions were also discussed in Ref. [19].

Next, we briefly discuss the many-body mechanisms included in the modification of the pion propagator in a nuclear medium. In cold nuclear matter, the pion spectral function exhibits a mixture of the pion quasiparticle mode and particle-hole $(\mathrm{ph})$, Delta-hole $(\Delta \mathrm{h})$ excitations. Following the calculation in Ref. [20] (extended to finite temperatures in Ref. [19]), the lowest-order irreducible $P$-wave pion selfenergy reads

$$
\begin{aligned}
& \Pi_{\pi N N^{-1}+\pi \Delta N^{-1}}^{p}\left(q_{0}, \vec{q} ; \rho, T\right) \\
& \quad=\left(\frac{f_{N}}{m_{\pi}}\right)^{2} \vec{q}^{2}\left[U_{N N^{-1}}\left(q_{0}, \vec{q} ; \rho, T\right)+U_{\Delta N^{-1}}\left(q_{0}, \vec{q} ; \rho, T\right)\right],
\end{aligned}
$$

where the finite temperature Lindhard functions for the ph and $\Delta \mathrm{h}$ excitations are given in detail in the appendix of Ref. [19]. The strength of the collective modes excited by the pion is further modified by repulsive, spin-isospin $N N$ and $N \Delta$ short-range correlations [21], which we include in a phenomenological way with a Landau-Migdal effective interaction.

At normal nuclear matter density, the pion spectral function clearly exhibits the different modes excited in the medium. At low momentum, the pion quasiparticle peak carries most of the strength together with a moderate contribution of the ph excitations at lower energies. The pion mode feels a sizable attraction with respect to that in free space. At larger momentum values of a few hundred $\mathrm{MeV} / c$, the excitation of the $\Delta \mathrm{h}$ mechanism takes over and provides a considerable amount of strength overlapping with the pion quasiparticle peak that broadens considerably.

At finite temperatures, the softening of the nucleon occupation number due to thermal motion causes a broadening of the three modes present in the spectral function. In the next section we discuss how these features of the pion spectral function reflect in the saturation of the different EWSRs.

\section{RESULTS AND DISCUSSION}

In this section we analyze the behavior of the energyweighted sum rules for the particular models of the kaon and pion properties in a hot and dense nuclear medium described in the former section. As commonly done [14-16], we depict the left-hand side of each sum rule as a function of the upper limit of the energy integration. This allows us to examine how relevant is the contribution of the different modes populating the meson spectral function (collective modes, quasiparticle peak) in saturating the sum rule. Note that depending on the energy weight of the sum rule different energy regions will be scanned. The horizontal scale ends at $1000 \mathrm{MeV}$ because, as noted in the previous section, the model calculation of the $K(\bar{K})$ self-energy cannot be trusted beyond this energy value.

The $m_{-1}, m_{0}^{(-)}$, and $m_{0}^{(+)}$sum rules for the antikaon propagator are shown in Fig. 1 in the case of normal nuclear matter density, zero temperature, and $150 \mathrm{MeV} / c$ kaon momentum. The contributions from $\bar{K}$ and $K$ to the left-hand side of the sum rule, cf. Eqs. (10), (11), and (16), are depicted

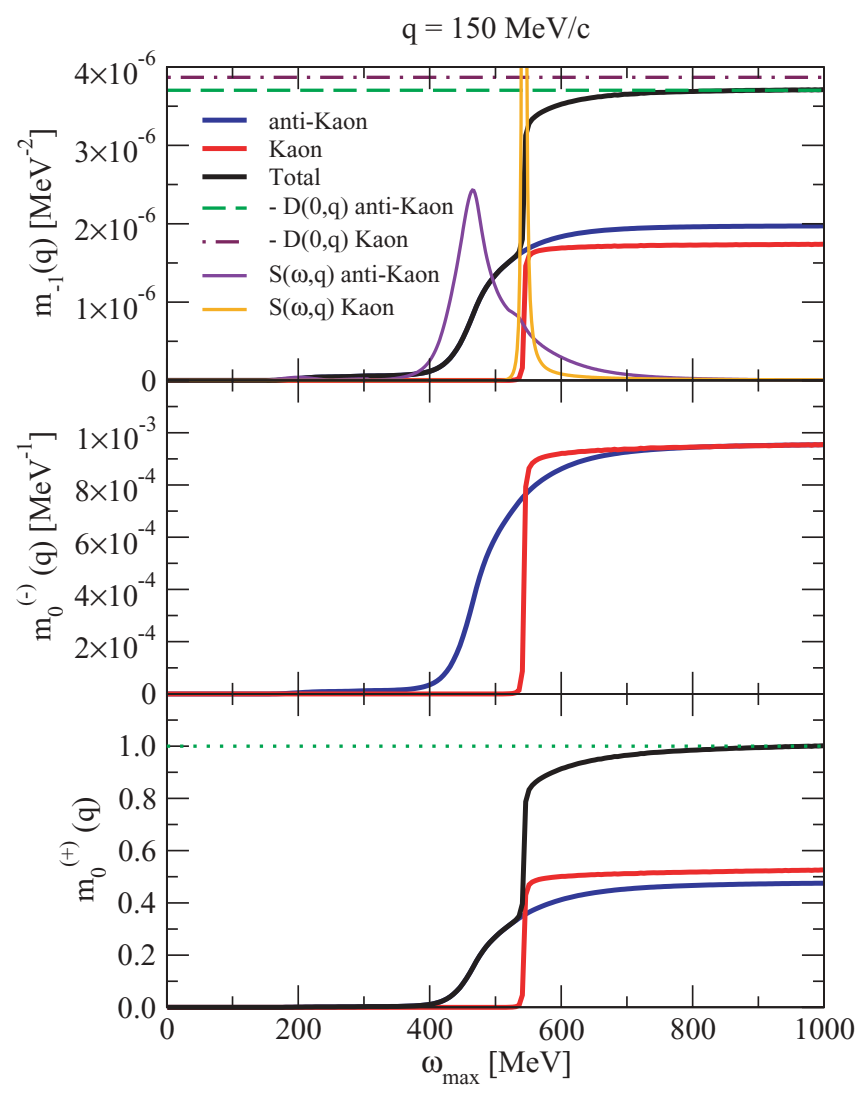

FIG. 1. (Color online) $m_{-1}, m_{0}^{(-)}$, and $m_{0}^{(+)}$sum rules for the $K$ and $\bar{K}$ spectral functions at $q=150 \mathrm{MeV} / c, \rho=\rho_{0}$ and zero temperature. The $\bar{K}, K$ spectral functions are also displayed for reference in arbitrary units. Note that the $m_{0}^{(+)}$sum rule (lower panel) is independent of the meson momentum. 
separately. The $\bar{K}$ and $K$ spectral functions are also shown for reference in arbitrary units.

The left-hand side of the $m_{-1}$ sum rule (upper panel) converges properly and saturates a few hundred $\mathrm{MeV}$ beyond the quasiparticle peak. The antikaon part has a soft behavior as the $\bar{K}$ spectral function spreads as a consequence of the mixing of the quasiparticle peak and the $\Lambda(1405) \mathrm{h}$ mode. Note that the subthreshold $P$-wave Yh components, although small at low momentum, have a visible contribution to the sum rule below the quasiparticle peak, as a consequence of the $\omega^{-1}$ energy weight in the integrand of Eq. (16). The $K$ contribution carries about half the weight of the saturated sum rule, as it would be the case in the absence of interactions. The contribution, which is mainly concentrated at the quasiparticle energy, reflects the narrowness of the $K$ spectral function even at normal matter density, as no baryonic resonances in the $S=+1$ channel can be excited.
We have also plotted in Fig. 1 the right-hand side of the $m_{-1}$ sum rule both for the antikaon and kaon, namely their off-shell propagators evaluated at zero energy (modulo a minus sign). The difference between both values indicates $\Pi_{K}\left(q^{0}=\right.$ $0, \vec{q} ; \rho) \neq \Pi_{\bar{K}}\left(q^{0}=0, \vec{q} ; \rho\right)$, which reflects the violation of crossing symmetry present in the chiral model employed for the kaon and antikaon self-energies. Although this model works well in the timelike region for kaon (antikaon) energies from $m_{K}$ to about $1 \mathrm{GeV}$, its limitations show up for spacelike kaons (antikaons) since their zero-mode propagators do not coincide.

We recall that, in fact, the chiral $K(\bar{K}) N$ amplitudes are dominated by the unitarized $S$-wave component that is built by neglecting the explicit exchange of a meson-baryon pair in a $t$-channel configuration, thus violating crossing symmetry. This approximation plays a minor role in the $S$-wave amplitudes at energies around the $K(\bar{K}) N$ threshold but may
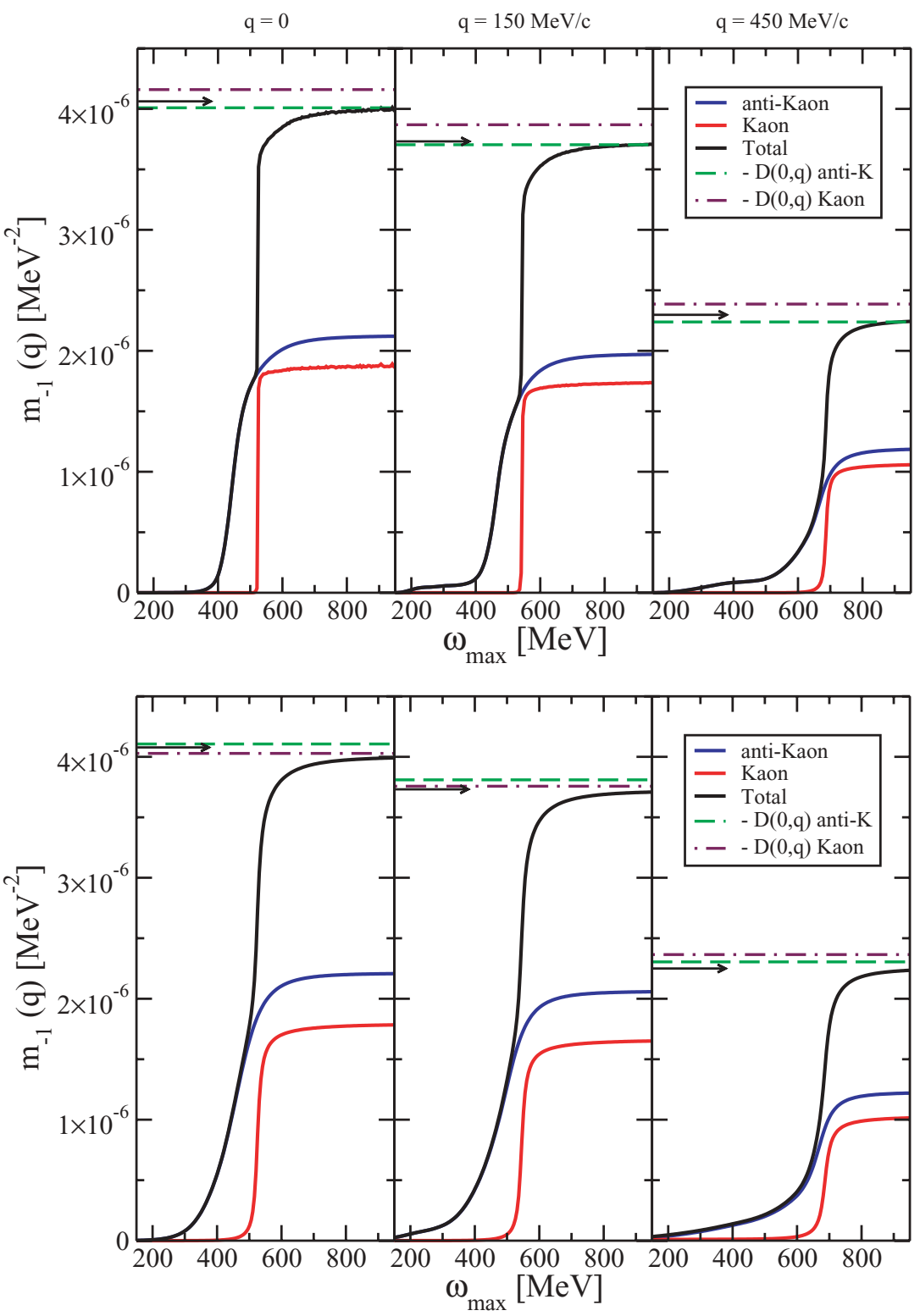

FIG. 2. (Color online) $m_{-1}$ sum rule for the $K$ and $\bar{K}$ spectral functions at several momenta $(q=0,150,450 \mathrm{MeV} / c)$ and $\rho=\rho_{0}$. (Upper panel) Zero temperature. (Lower panel) $T=$ $100 \mathrm{MeV}$. The arrows indicate the value of the right-hand side in vacuum. 
turn relevant for the largely off-shell amplitudes explored in the evaluation of the $K(\bar{K})$ propagator at $q^{0}=0 \mathrm{MeV}$. With this in mind, we may still expect the saturated value of the left-hand side of the $m_{-1}$ sum rule to provide a constraint for the value of the zero-mode propagator appearing on the right-hand side. This is so because, as seen in Fig. 1, the very low energy contribution to the saturation value of the left-hand side of the sum rule is marginal, whereas most of the strength sets in at energies of the order of the meson mass, where the neglected terms of the $K(\bar{K}) N$ amplitudes are irrelevant. Therefore, the fact that the sum rule is well satisfied when comparing the left-hand side with $D_{\bar{K}}(0, \vec{q})$ indicates that neglecting the $t$-channel dynamics in the $\bar{K} N$ interaction is actually quite a good approximation. In fact, the omitted mechanisms start appearing at the one-loop level and involve the excitation of intermediate $K N$ states in a $t$-channel configuration. These $K N$ loop contributions have shown to be relatively weak in the dynamics of the crossed $s$-channel configuration of the $K N$ system. Conversely, the neglected $t$-channel meson-baryon loop terms in the $K N$-scattering amplitude involve the excitation of both $S=-1, \bar{K} N$, and $\pi Y$, intermediate states, which have been shown to interact quite strongly in the $s$-channel configuration present in $\bar{K} N$ dynamics. It is then clear that the calculated $\bar{K}$ propagator is more accurate because the neglected terms are smaller, a fact that is corroborated by the better fulfillment of the $m_{-1}$ sum rule in this case.

The $m_{0}^{(-)}$sum rule tells us that the areas subtended by the $K$ and $\bar{K}$ spectral functions should coincide. This is indeed the case for the calculation considered here, as can be seen in the middle panel of Fig. 1. We emphasize here that the fulfillment of the $m_{0}^{(-)}$sum rule for the model of kaon interactions under analysis is far from being trivial. We recall that, whereas one expects the $\bar{K}$ and $K$ spectral functions to be related by the retardation property, $S_{\bar{K}}(-\omega)=-S_{K}(\omega)$, the actual calculation of the meson self-energies is done exclusively for positive meson energies [timelike region in the $\bar{K}(K) N$ scattering amplitude]. The analytical constraints are nevertheless imposed in the self-consistent evaluation of the scattering amplitudes and self-energies [19] through the use of $\bar{K}$ and $K$ in-medium propagators in the form of Eq. (2), which couples the information of the two spectral functions. We note, for instance, that a simplified mean-field-like description of the meson self-energies by means of effective in-medium masses, namely $S(\omega, \vec{q})=\delta\left[\omega-\omega^{*}(\vec{q})\right] / 2 \omega^{*}(\vec{q})$ with $\omega^{*}(\vec{q})=\sqrt{\vec{q}^{2}+m^{* 2}}$, would clearly violate the $m_{0}^{(-)}$sum rule because $\Delta m_{\bar{K}}^{*}(\rho)<0$ and $\Delta m_{K}^{*}(\rho)>0$.

The $m_{0}^{(+)}$sum rule saturates to one independently of the meson momentum, nuclear density or temperature, thus posing a strong constraint on the accuracy of the calculations. It has been thoroughly used to test the quality of the nucleon spectral function in the nuclear many-body problem. The lower panel in Fig. 1 shows that the calculated $K$ and $\bar{K}$ spectral functions fulfill this sum rule to a high precision. The particle and antiparticle parts converge to different values in general, but the sum perfectly saturates to the required value of 1 . Also note that saturation is progressively shifted to higher energies as we examine sum rules involving higher-order weights in energy.
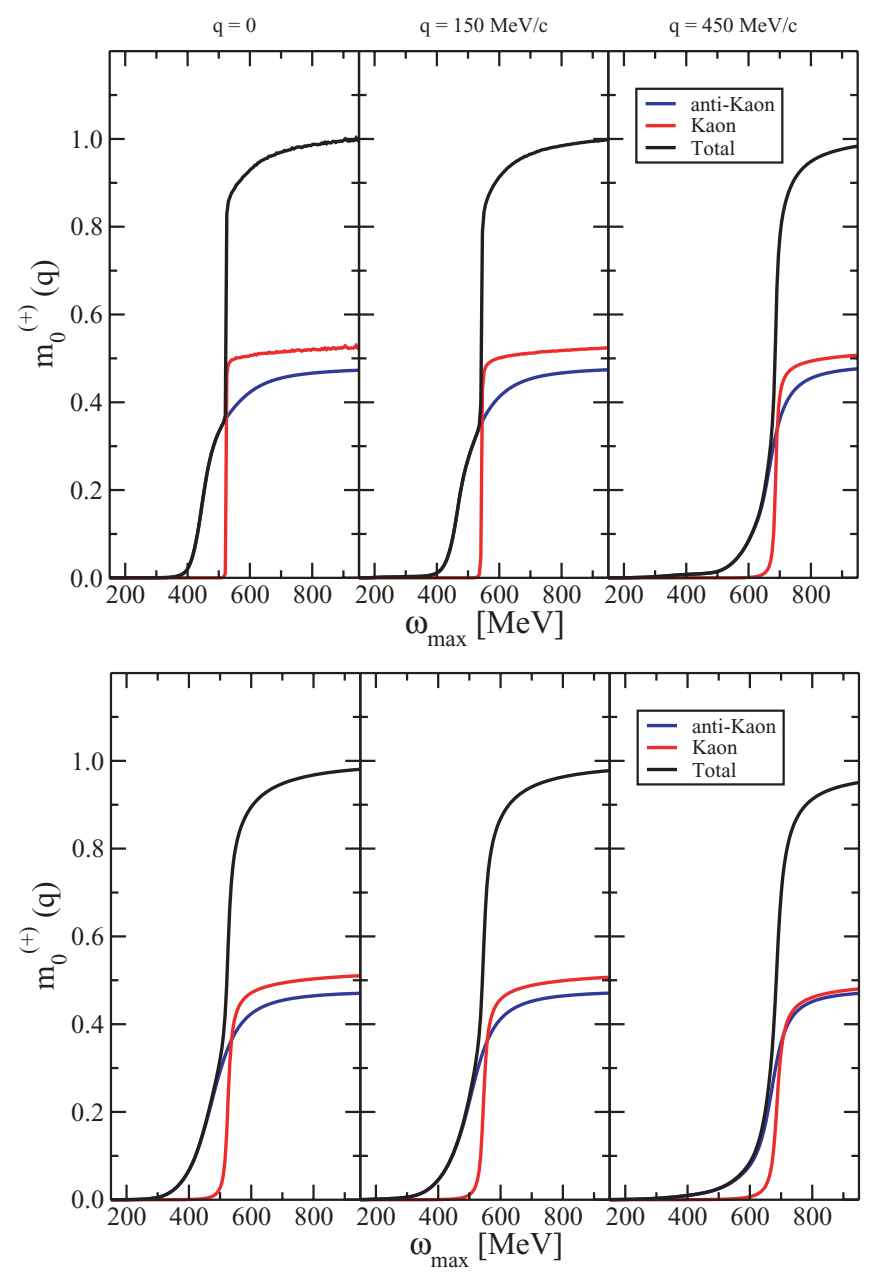

FIG. 3. (Color online) $m_{0}^{(+)}$sum rule for the $K$ and $\bar{K}$ spectral functions at several momenta $(q=0,150,450 \mathrm{MeV} / c)$ and $\rho=\rho_{0}$. (Upper panels) Zero temperature. (Lower panels) $T=100 \mathrm{MeV}$.

Next we show in Figs. 2 and 3 the results for $m_{-1}$ and $m_{0}^{(+)}$, respectively, at normal nuclear density for different kaon momenta, at zero temperature (upper panels) and $T=100 \mathrm{MeV}$ (lower panels). As the meson momentum is increased, the saturation of the integral part of the sum rules is progressively shifted to higher energies, following the strength of the spectral distribution. In particular, $m_{-1}$ exhibits a growing sensitivity to the low-energy $P$-wave $\mathrm{Yh}$ modes, which are enhanced at finite momentum. At finite temperature the $\bar{K}$ spectral function spreads considerably [19] and in particular acquires a sizable low-energy tail from smearing of the Fermi surface, which contributes substantially to the left-hand side of the sum rule below the quasiparticle peak. Note also that the $K$ contribution softens at finite temperature and increasing momenta, as the $K$ in-medium decay width is basically driven by the $K N$ thermal phase space.

We observe that the $m_{-1}$ sum rule is well satisfied by the zero-temperature $\bar{K}$ spectral function for the three different momenta represented in the plot, which reinforces our discussion about the model approximations elaborated above (we have also checked the momentum dependence of 
the saturated sum rule in a wide range of momenta from 0 to $1 \mathrm{GeV} / c$ with the same conclusions). At finite temperature, however, there is no longer a good agreement for the antikaon. This is not a failure of the model interaction but of the calculation itself: the expression in Eq. (30) for the (dominant) $S$-wave antikaon self-energy contains in fact an approximation to the dispersive contribution, as explained in Appendix B of Ref. [19], which is appropriate for energies close to the kaon mass or higher. It can also be seen there that the dispersive contributions to the kaon and antikaon self-energy practically vanish at $q^{0}=0$ (this is exact for the imaginary part of the self-energy), while our approximated finite- $T$ expression, Eq. (30), does not satisfy this requirement. Therefore, it is expectable that neither the kaon nor the antikaon self-energies fulfill properly the $m_{-1}$ sum rule at finite $T$ under the current approximations. Moreover, we observe that the discrepancy of either contribution with the left-hand side is of the same order of magnitude as if we had just used the free propagator to evaluate the right-hand side. As a consequence, one may not employ this sum rule as a way to test the interaction model, unless a very refined finite- $T$ calculation of the self-energy is performed in the far off-shell, spacelike energy region.

The $m_{0}^{(+)}$sum rule is fulfilled satisfactorily for the different momenta and the two temperatures considered. We note, though, that convergence turns slower for increasing momentum and finite temperatures and, in some of the cases shown and up to the maximum energy explored, the limiting value of one has not yet been reached.

We have checked that, for a wide range of momentum values, the $m_{0}^{(-)}$sum rule at $T=0$ converges to zero with similar precision as the $q=150 \mathrm{MeV} / c$ case shown in Fig. 1 . At finite $T$, the agreement is slightly worse but consistent with zero, admitting a $3 \%$ error in the calculated spectral functions.
In the case of the $m_{1}^{(-)}$sum rule, the additional $\omega^{2}$ weight tends to magnify the numerical inaccuracies or model deficiencies of the spectral functions. Nevertheless, for any of the two temperatures explored in this work, we find that the finite value at which the $m_{1}^{(-)}$sum rule saturates is compatible with zero if one admits a $10 \%$ numerical error in our calculated spectral function at energies around $1 \mathrm{GeV}$. Finally, we do not evaluate the $m_{1}^{(+)}$sum rule for our model calculation of the kaon spectral functions. The reason is twofold: on the one hand, the $K(\bar{K})$ spectral function has been calculated only up to about $1 \mathrm{GeV}$, due to limitations in the validity of the chiral unitary amplitudes, while the $m_{1}^{(+)}$sum rule carries a $\omega^{3}$ energy weight and thus the contribution of higher energies is still relevant in establishing the saturation of the left-hand side. On the other hand, the nondispersive contribution of the self-energy, associated to the high energy limit of the interaction and entering the right-hand side, corresponds in our model to the tree-level $\bar{K}(K) N$ vertex from the meson-baryon chiral Lagrangian. Its essentially linear energy dependence cannot be extrapolated to high energies without introducing hadronic form factors (and thus additional free parameters), leaving the actual value of $\Pi_{\bar{K}(K)}^{\infty}$ unconstrained.

The pion sum rules are discussed in the following. They exhibit notable differences with respect to those of the kaon propagator. To start with, there are no sum rules weighted with even energy powers, so we shall present results on $m_{-1}, m_{0}$, and $m_{1}$. Second, the pion spectral function at intermediate momenta displays well-separated collective modes, particularly at very low energies, which can be easily tracked in the sum rule saturation. The changes introduced by finite temperature are also visible and worth discussing.

In Fig. 4 (left column) we show $m_{-1}$ for two pion momenta, at normal nuclear density and zero temperature. In the case of

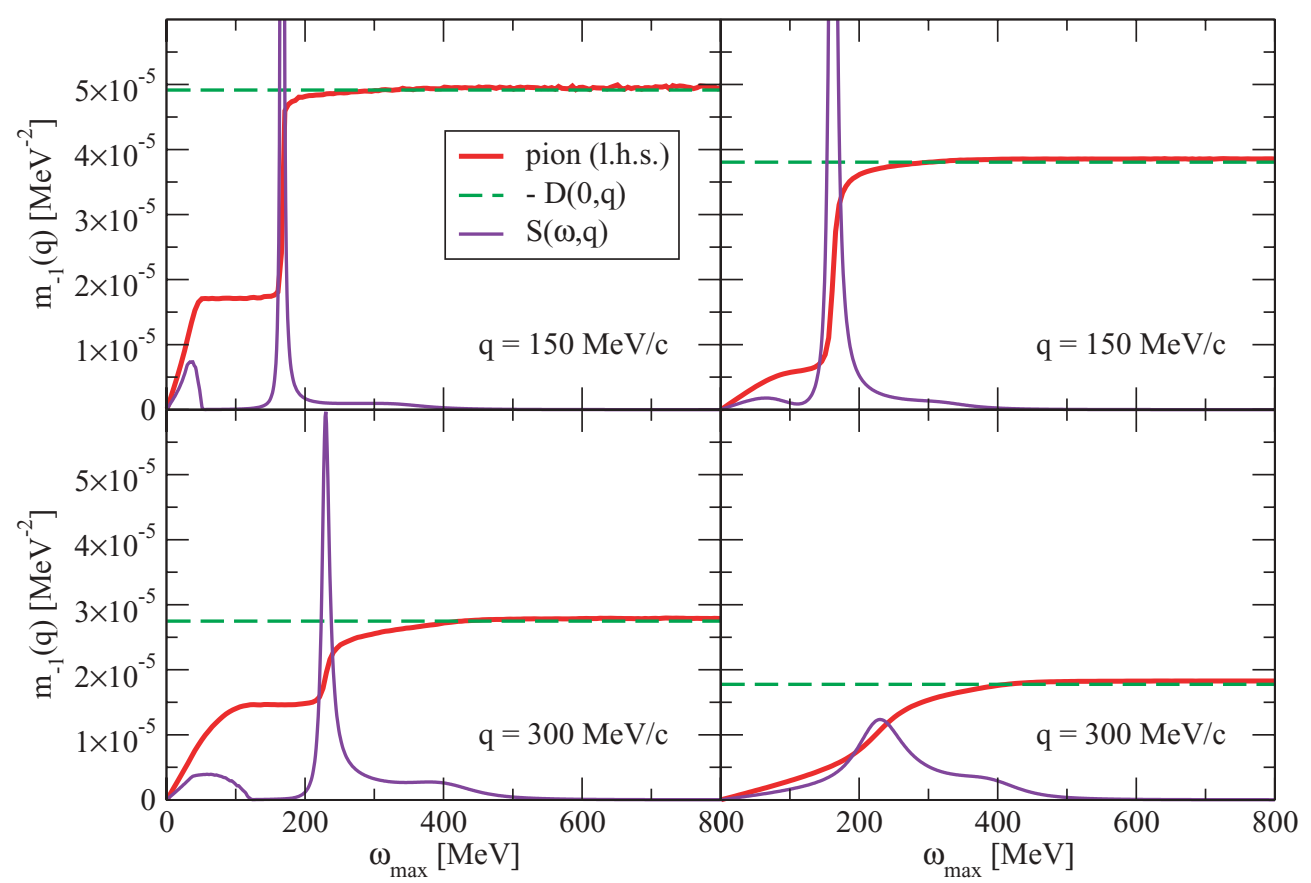

FIG. 4. (Color online) $m_{-1}$ sum rule for the pion spectral function at $q=150,300 \mathrm{MeV} / c$ and $\rho=\rho_{0}$. The left column corresponds to the zero temperature result and the right column to $T=100 \mathrm{MeV}$. The pion spectral function is also displayed for reference in arbitrary units. 
$q=150 \mathrm{MeV} / c$ most of the strength of the spectral function is carried by the pion quasiparticle peak. However, the ph component lies at low energies and therefore is rather enhanced in the integral by the inverse energy weight, contributing in more that $30 \%$ to the saturation value, whereas the pion quasiparticle peak practically carries the remaining strength. The $\Delta \mathrm{h}$ mode is barely visible to the right-hand side of the pion peak and contributes little at small momentum. Note the plateau in $m_{-1}$ due to the energy gap between the low-energy part of the spectrum and the pion mode. At higher momentum $(q=300 \mathrm{MeV} / c)$, the ph and $\Delta \mathrm{h}$ excitations acquire more relevance with respect to the quasipion. The left-hand side saturates and is in good agreement with the right-hand side, slightly overshooting the value of the inverse pion propagator at zero energy for increasing momentum. We understand these tiny deviations as originating by the implementation of the $\Delta$ decay width in the $\Delta \mathrm{h}$ contribution, which may lead to small violations of the analytical properties of the pion self-energy and propagator. Essentially, the $\Delta$ self-energy employed here accounts for the $\Delta$ width and its energy dependence coming from its decay to a $\pi N$ pair in $P$ wave. However, the nucleon momentum in the $\Delta \mathrm{h}$ excitation is averaged out to make the $\Delta$ self-energy dependent only on the pion external four-momentum $q$. The fulfillment of the $m_{-1}$ sum rule therefore indicates that, up to tiny deviations tied to these kinematical averages, accounting for the $\Delta$ decay width and its energy dependence not only provides a more realistic description of the phenomenology but additionally complies with analyticity requirements through the sum rules. As a test, we have also calculated $m_{-1}$ with a constant $\Delta$ decay width and the agreement between left-hand side and right-hand side of the sum rule is far worse than in the present model.

At finite temperature, the softening of the nucleon occupation number due to thermal motion causes the broadening of the three modes, as can be seen in the right column of Fig. 4. At a momentum value of $300 \mathrm{MeV} / c$ they are rather mixed, which removes the plateau visible at zero temperature (left panels), and the contribution from each excitation mechanism to the left-hand side of the sum rule can no longer be resolved.

The $m_{0}$ sum rule is depicted in Fig. 5 for three values of the pion momenta and $T=0,100 \mathrm{MeV}$. Despite the markedly different distribution of strength in the spectral density with increasing momentum and temperature, the sum rule is quite well satisfied in all cases. The deviations observed may also be attributed to the small violations of the analytical properties of the pion self-energy mentioned above.

Finally we show $m_{1}$ for the pion propagator in Fig. 6, for several momenta and two temperatures, $T=0$ and $100 \mathrm{MeV}$. The $m_{1}$ sum rule carries a $\omega^{3}$ energy weight that makes it sensitive to higher energies and thus its convergence is much slower. Conversely, the contribution from low-energy modes is marginal in this case. The pion self-energy analyzed here, built from the tree-level $P$-wave coupling to $\mathrm{ph}$ and $\Delta \mathrm{h}$ modes, admits a purely dispersive representation, as it can be easily derived from the Lindhard function. Therefore, $\Pi_{\pi}^{\infty}=0$ in our model and the left-hand side of the sum rule is seen to slowly converge to the squared single-particle pion energy in vacuum, $\omega_{\pi}^{2}=m_{\pi}^{2}+\vec{q}^{2}$.
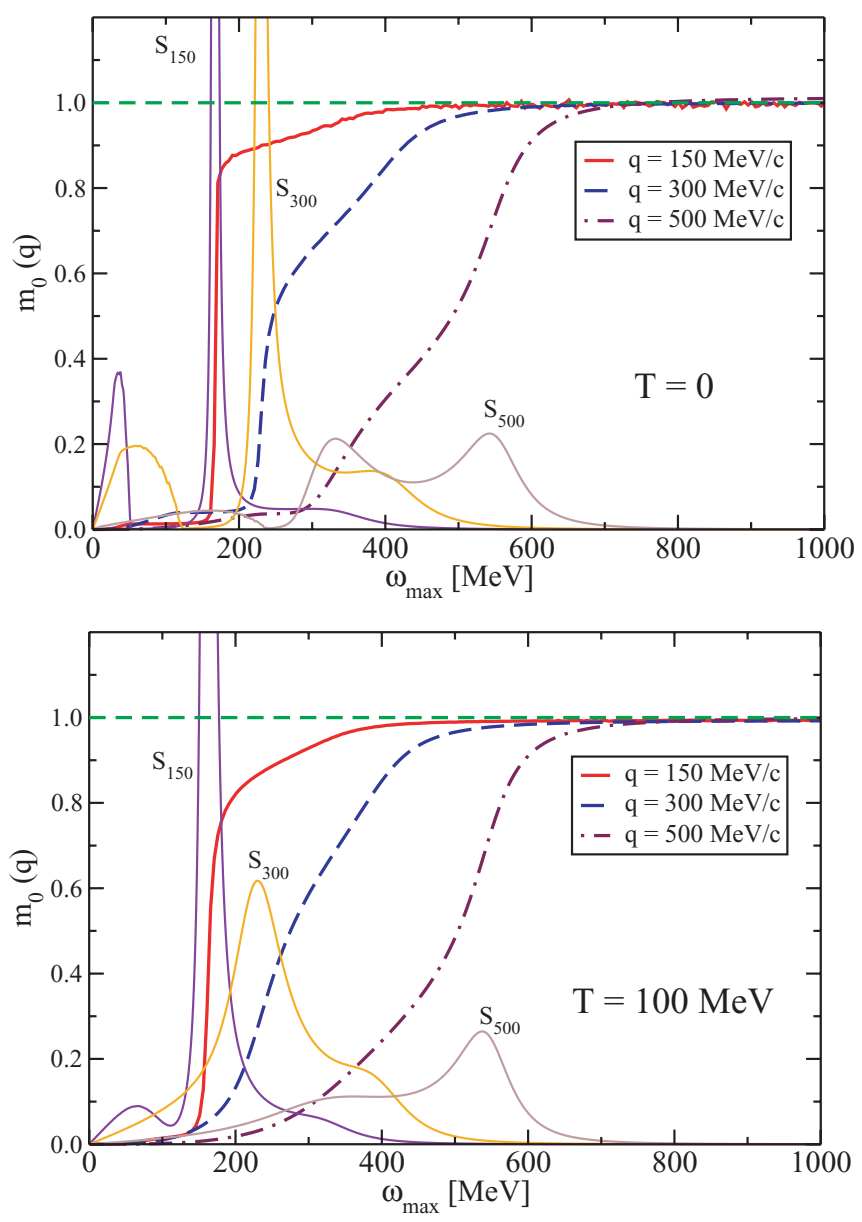

FIG. 5. (Color online) $m_{0}$ sum rule for the pion spectral function at several momenta, $\rho=\rho_{0}$ and $T=0,100 \mathrm{MeV}$. The pion spectral functions are also displayed for reference in arbitrary units, labeled as $S_{q}$ with $q$ the corresponding momentum in $\mathrm{MeV} / c$. Note that this sum rule is independent of the meson momentum.

\section{SUMMARY AND OUTLOOK}

In summary, we have presented a derivation of the energyweighted sum rules of the meson propagator in nuclear matter, which can be applied to a wide range of scenarios such as meson systems with a different in-medium behavior of particle and antiparticle modes, isospin-asymmetric matter, and matter at finite temperature. We have particularized the sum rules for kaons and pions in cold and hot symmetric nuclear matter, where specific models for the meson self-energy and spectral function have been analyzed from the point of view of the saturation of the sum rules.

The sum rules have been shown to be a useful tool to magnify troublesome situations where certain approximations typically done in the calculation of the scattering amplitudes (and, thus, of the meson self-energies) may not work properly in particular kinematical regimes. This is possible because the sum rules explored relate the energy-weighted meson spectral function, integrated over all energies, to the meson propagator, evaluated at low or high energies, as well as to model-independent quantities. For instance, thanks to visible 

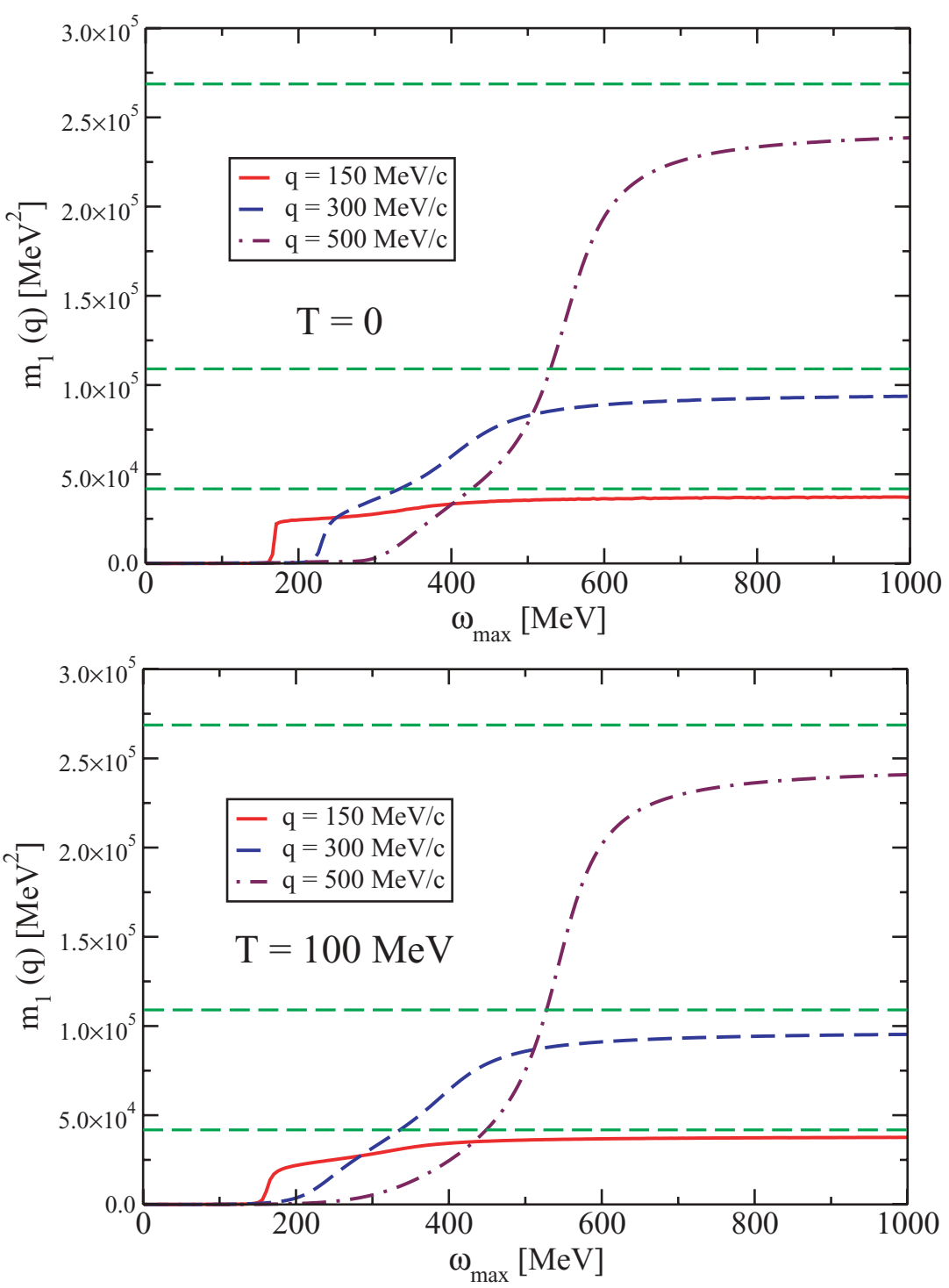

FIG. 6. (Color online) $m_{1}$ sum rule for the pion spectral function at several momenta, $\rho=$ $\rho_{0}$ and $T=0,100 \mathrm{MeV}$. The dashed, horizontal lines correspond to the right-hand side of the sum rule for each momentum, $\omega_{\pi}^{2}=m_{\pi}^{2}+\vec{q}^{2}$. deviations in the $m_{-1}$ sum rule, we have seen that violation of crossing symmetry in the chiral unitary $K(\bar{K}) N$ interaction model employed becomes relevant at timelike energies. The $m_{-1}$ sum rule is not properly fulfilled in the case of pions either if a constant (energy-independent) $\Delta$ decay width is employed in the intermediate $\Delta \mathrm{h}$ excitations. An oversimplified description of medium effects on the meson single-particle properties, such as the use of effective in-medium masses or approximating meson spectral functions by quasiparticle Breit-Wigner peaks - thus ignoring the role of $\mathrm{Nh}, \Delta \mathrm{h}, \mathrm{Yh}$, or $\mathrm{Y}^{*} \mathrm{~h}$ components-may lead to violations of the sum rules already at the lowest orders. We also note that, even if the interaction model fulfilled the proper analyticity requirements, certain sum rules may also be useful to check the accuracy of the numerical evaluation of the meson spectral functions, as is the case of the momentum-independent $m_{0}$.

The present work can be used to study the quality of manybody approaches and interaction models in other systems such as light vector and axial-vector meson resonances, where a straightforward extension of the formalism is required to describe transverse and longitudinal modes. The study of open- and hidden-charm meson resonances in hot and dense matter has also received much interest lately and will be subject of experimental investigation in heavy-ion experiments at Facility for Antiproton and Ion Research. Present and future calculations of the interaction of these systems with the medium can also be scrutinized from the point of view of EWSR's. Work along these lines is in progress.

\section{ACKNOWLEDGMENTS}

This work is partly supported by the EU Contract No. MRTN-CT-2006-035482 (FLAVIAnet), by the Contracts FIS2008-01661/FIS and FPA2008-00592 from MICINN (Spain), by the Generalitat the Catalunya Contract 2009SGR-1289, and by the UCM-BSCH Contract GR58/08 910309. We acknowledge the support of the European Community-Research Infrastructure Integrating Activity "Study of Strongly Interacting Matter" (HadronPhysics2, Grant agreement No. 227431) under the Seventh Framework Programme of EU, the "RFF-Open and Hidden Charm at PANDA" project from the Rosalind Franklin Programme of 
the University of Groningen and the Helmholtz International Center for FAIR within the framework of the LOEWE program (Landesoffensive zur Entwicklung Wissenschaftlich-
Ökonomischer Exzellenz) launched by the State of Hesse (Germany). D. Cabrera acknowledges support from the "Juan de la Cierva" Programme (MICINN, Spain).
[1] R. Rapp and J. Wambach, Adv. Nucl. Phys. 25, 1 (2000).

[2] C. Fuchs, Prog. Part. Nucl. Phys. 56, 1 (2006).

[3] E. Friedman and A. Gal, Phys. Rep. 452, 89 (2007).

[4] R. S. Hayano and T. Hatsuda, arXiv:0812.1702 [nucl-ex].

[5] http://www.gsi.de/fair/; http://www.gsi.de/fair/experiments/CBM/index_e.html; http://www-panda.gsi.de/auto/home.htm; http://www.gsi.de/fair/EU-Construction/HADES.html; http://www.gsi.de/forschung/kp/kp1/experimente/fopi/.

[6] E. Lipparini and S. Stringari, Phys. Rep. 175, 103 (1989).

[7] H. R. Glyde, Excitations in Liquid and Solid Helium (Clarendon Press, Oxford, 1994).

[8] F. Dalfovo, S. Giorgini, L. Pitaevskii, and S. Strigari, Rev. Mod. Phys. 71, 463 (1999).

[9] S. Weinberg, Phys. Rev. Lett. 18, 507 (1967).

[10] J. I. Kapusta and E. V. Shuryak, Phys. Rev. D 49, 4694 (1994).

[11] Y. Kim, R. Rapp, G. E. Brown, and M. Rho, Phys. Rev. C 62, 015202 (2000).

[12] D. Cabrera, D. Jido, R. Rapp, and L. Roca (in preparation).
[13] J. P. Blaizot and G. Ripka, Quantum Theory of Finite Systems (MIT Press, Cambridge, MA, 1985).

[14] A. Polls, A. Ramos, J. Ventura, S. Amari, and W. H. Dickhoff, Phys. Rev. C 49, 3050 (1994).

[15] T. Frick, H. Muther, and A. Polls, Phys. Rev. C 69, 054305 (2004).

[16] A. Rios, A. Polls, and H. Muther, Phys. Rev. C 73, 024305 (2006).

[17] A. K. Dutt-Mazumder, Nucl. Phys. A713, 119 (2003).

[18] L. Tolos, A. Ramos, and E. Oset, Phys. Rev. C 74, 015203 (2006).

[19] L. Tolos, D. Cabrera, and A. Ramos, Phys. Rev. C 78, 045205 (2008).

[20] E. Oset, P. Fernandez de Cordoba, L. L. Salcedo, and R. Brockmann, Phys. Rep. 188, 79 (1990).

[21] E. Oset, H. Toki, and W. Weise, Phys. Rep. 83, 281 (1982).

[22] A. L. Fetter and J. D. Walecka, Quantum Theory of ManyParticle Systems (Courier Dover, New York, 2003). 